\title{
Correction to: Role of Amino Acid Metabolism in the Virulence of Human Pathogenic Fungi
}

\author{
Enrico Garbe ${ }^{1,2} \cdot$ Slavena Vylkova ${ }^{1,2}$
}

Published online: 9 August 2019

(C) Springer Nature Switzerland AG 2019

\section{Correction to: Current Clinical Microbiology Reports https://doi.org/10.1007/s40588-019-00124-5}

The author found a mistake in their published article. Some of the requested corrections during the proofreading of the article were not addressed. These corrections are:

- Affiliation for Enrico Garbe: affiliation 2 is not indicated at the name

- Affiliation 2 was changed to Septomics Research Centre, Leibniz Institute for Natural Product Research and Infection Biology - Hans Knoell Institute, Jena, Germany

- Table 1: In "Phenotype C. albicans": ssy16 $\Delta$ changed to ssy $1 \Delta$ and reference list $[20,26,51]$, reference 51 was taken out and \#20 was changed to \#25; ssy $1 \Delta$ italicized to $\operatorname{ssy} 1 \Delta$

In "Phenotype C. neoformans," reference \#46 was put in brackets [], text for CnAap1-8 phenotype changed to aap $4 \Delta /$ aap $5 \Delta$, aap $1 \Delta /$ aap $2 \Delta$, and aap $8 \Delta$ strains are attenuated in G. melonella model and aap $4 \Delta /$ aap $5 \Delta$ in murine inhalation model $[34,35]$

- Third page: A "S. cerevisiae" was italicized to "S. cerevisiae"

- Fifth page: "Stimulus" changed to "stimulus".

The original article has been corrected.

Publisher's Note Springer Nature remains neutral with regard to jurisdictional claims in published maps and institutional affiliations.

The online version of the original article can be found at https://doi.org/ 10.1007/s40588-019-00124-5

Slavena Vylkova

slavena.vylkova@leibniz-hki.de

1 NWG Host Fungal Interfaces, Friedrich Schiller University, Jena, Germany

2 Septomics Research Centre, Leibniz Institute for Natural Product Research and Infection Biology-Hans Knoell Institute, Jena, Germany 